\title{
New beam-tracking simulation code using bulk-to-point calculation technique for space charge fields
}

\author{
A. Mizuno* \\ JASRI/SPring-8, 1-1-1 Koto, Sayo, Hyogo 679-5198, Japan
}

(Received 11 December 2015; published 29 February 2016)

\begin{abstract}
A new two-dimensional beam-tracking simulation code for electron injectors using a bulk-to-point calculation technique for space charge fields was developed. The calculated space charge fields are produced not by a point charge but by a hollow cylinder that has a volume. Each tracked electron is a point charge. This bulk-to-point calculation technique for space charge fields is based on that used in the multiple beam envelope equations, which were developed by the author. The multiple beam envelope equations are a set of differential equations for investigating the beam dynamics of electron injectors and can be used to calculate bunched beam dynamics with high accuracy. However, there is one limitation. The bunched beam is assumed to be an ensemble of several segmentation pieces in both the transverse and longitudinal directions. In this bunch model, each longitudinal segmentation slice in a bunch must not warp; consequently, the accuracy of the calculated emittance is reduced in the case of a highly charged beam for calculations of a typical rf gun injector system. This limitation is related to the calculation model of longitudinal space charge fields. In the newly developed beam-tracking simulation code, the space charge field calculation scheme is upgraded and the limitation has been overcome. Therefore, the applicable range is extended while maintaining the high accuracy of emittance calculations. Simultaneously, the calculation time is markedly shortened because the emittance dependence on the segmentation number is extremely weak. In this paper, several examples of beam dynamics that cannot be calculated accurately using the multiple beam envelope equations are demonstrated using the new beam-tracking simulation code. The accuracy of the calculated emittance is also discussed.
\end{abstract}

DOI: 10.1103/PhysRevAccelBeams.19.024201

\section{INTRODUCTION}

The emittance calculation technique is important in the design of electron injectors, particularly very low emittance electron sources such as X-ray free-electron lasers. There have been many analytical solutions [1-4] for beam dynamics, although it is difficult to accurately calculate practical bunch shapes and detailed emittance behavior. On the other hand, particle-tracking simulation codes [5-9] are useful for calculating the dynamics of complex bunch shapes of practical beams. However, the calculated emittances often depend on the number of particles.

These dependences are often caused by the calculation scheme of the space charge field which is produced by a point charge. This scheme makes calculation noise larger. Calculation techniques for space charge fields produced not by a point charge but by a bulk charge have been investigated [10-12]. In Ref. [10], the semianalytical calculation for short bunched electron beam dynamics is accomplished. The bunched beam is divided longitudinally

*mizuno@spring8.or.jp

Published by the American Physical Society under the terms of the Creative Commons Attribution 3.0 License. Further distribution of this work must maintain attribution to the author $(s)$ and the published article's title, journal citation, and DOI. into several slices. Traces of each slice are solved by beam envelope equations with calculating space charge fields produced by each slice. In this code, however, transverse nonlinearity of the space charge fields cannot be calculated.

For accurate emittance calculations of short bunched electron beam dynamics, the author also developed a semianalytical solution $[13,14]$, which was proposed in 2012 , by combining an analytical method and a simulation method using a set of beam envelope equations with the calculation technique for the space charge field produced by a bulk charge. This method is referred to as "the multiple beam envelope equations" in this article.

In this method, a short bunched electron beam is assumed to be an ensemble of several segmentation pieces in both the transverse and longitudinal directions. The trajectory of each electron, which is a point charge and located at each segmentation corner, is solved using a beam envelope equation. The shape of the entire bunch is consequently calculated; thus, the emittances are successfully calculated from weighted mean values of the solutions for each obtained electron trajectory. When calculating space charge fields, they are assumed not to be produced by a point charge but by a longitudinal slice that has a volume, which means that the envelope equations include analytical expressions of space charge fields produced by the slice. These calculation techniques, that is, the code is 
semianalytical and the bunch is divided into several slices, are similar to those used in Ref. [10]. The different points are that the bunch is also divided into transversely and nonlinearity of transverse fields can be calculated in this method. Therefore, highly accurate emittance calculations can be performed.

In this method, however, there is one limitation with regard to an assumption on the segmentation pieces. Each longitudinal slice, which is perpendicular to the beam axis, is rigid and must not warp [13]. This assumption is caused by the model used to calculate space charge fields. That is, the longitudinal space charge fields at off-axis positions are assumed to be equal to the on-axis fields in the multiple beam envelope equations in order to avoid complicated forms of differential equations. However, the fields at offaxis positions are usually weaker than those at on-axis positions. Therefore, the shape of the bunch cannot be calculated accurately in the cases of highly charged beams.

To remove this limitation, the author has developed a new two-dimensional beam-tracking simulation code. The longitudinal space charge fields at off-axis positions are numerically calculated in advance and prepared as a data file. In the beam-tracking calculations, the longitudinal fields are referred to the data file by loading the file into the calculation process. Therefore, accurate and rapid calculations can be realized using the new code.

In Sec. II, the calculation scheme of the multiple beam envelope equations is summarized. In Sec. III, new features of the new beam-tracking simulation code are described. In Sec. IV, examples that cannot be accurately calculated using the multiple beam envelope equations are calculated using the new beam-tracking simulation code. They are the beam dynamics in the BNL-type rf gun cavity with high charge and those in the SPring-8 $\mathrm{rf}$ gun injector system with beam focusing. The accuracy of the calculated emittance in the new beam-tracking simulation code is also discussed.

\section{CALCULATION SCHEME FOR MULTIPLE BEAM ENVELOPE EQUATIONS}

The calculation scheme for the multiple beam envelope equations is summarized here. Figure 1 shows the bunch segmentation model used for the multiple beam envelope equations. The bunch is divided into $m$ slices in the longitudinal direction and $n$ parts in the transverse direction. Each electron is located at each segmentation corner and traced using the beam envelope equations. The trajectory of each electron at $z_{j}(j=1, \ldots, m+1)$ on the $z$-axis is calculated using the longitudinal envelope equation. The transverse trajectories of the electron located on the circumference of each segmentation boundary, $R_{j}(j=1, \ldots, m+1)$ and the electron located at $r_{i j}(i=1, \ldots, n$ and $j=1, \ldots, m+1)$, which is inside the bunch, are calculated using the transverse envelope equations. The longitudinal trajectories of the electrons at $R_{j}$ and $r_{i j}$ are assumed to be equal to the trajectory of the

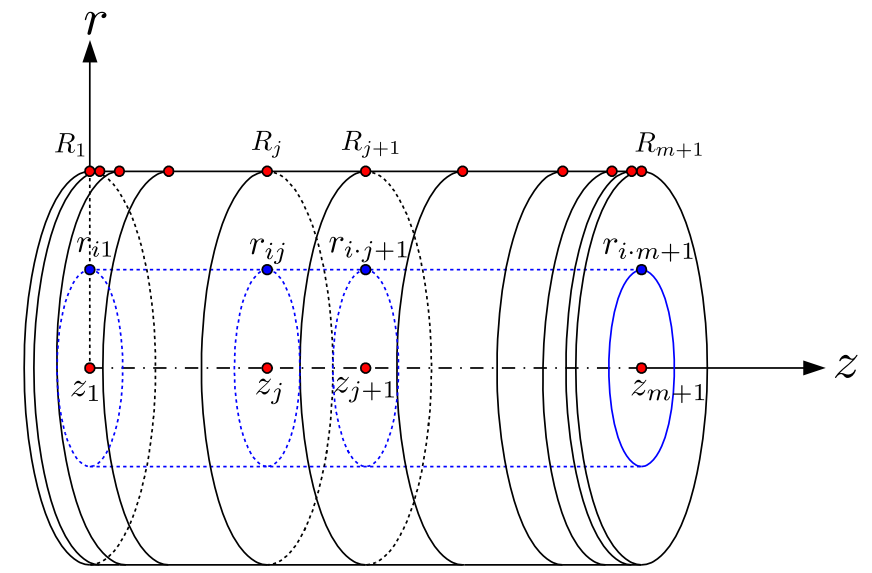

FIG. 1. Bunch segmentation model used for multiple beam envelope equations. This figure shows an initial bunch configuration.

electron at $z_{j}$ in order to avoid calculations of longitudinal space charge fields at the off-axis positions since these calculations are too complicated to form the differential equations. Therefore, each longitudinal slice is rigid and must not warp. $\beta_{j}$ is also defined as the normalized longitudinal velocity of each electron having suffix $j$ for differential equations with respect to electron energy. As a result, the trajectories are calculated by a set of simultaneous differential equations, which contain the $(n+3)(m+1)$ independent variables $R_{j}, r_{i j}, z_{j}$ and $\beta_{j}$ $(i=1, \ldots, n$ and $j=1, \ldots, m+1)$. In the rest of the paper, $m$ and $n$ are referred to as "segmentation numbers."

The longitudinal space charge field applied to each electron located at $z_{j}$ is the sum of the fields produced from each slice, which is enclosed by two disks with radii $R_{j}$ and $R_{j+1},(j=1, \ldots, m)$. The longitudinal field from each slice can be calculated analytically by integrating space charge fields produced by charges in a small volume over $\theta, r$ and $z:$ [13]

$$
\begin{aligned}
E_{z \text { slice }}(z)= & \frac{Q}{2 \pi \epsilon_{0} R_{s}^{2} L}\left(-\sqrt{\left(z-\frac{L}{2}\right)^{2}+\frac{R_{s}^{2}}{\gamma^{2}}}\right. \\
& \left.+\sqrt{\left(z+\frac{L}{2}\right)^{2}+\frac{R_{s}^{2}}{\gamma^{2}}}+\left|z-\frac{L}{2}\right|-\left|z+\frac{L}{2}\right|\right),
\end{aligned}
$$

where $E_{z \text { slice }}$ is the longitudinal space charge field, $Q$ is the charge of the slice, $R_{s}=\left(R_{j}+R_{j+1}\right) / 2$ is the radius of the slice, $L$ is the longitudinal width of the slice, $z$ is the distance from the center of the slice to the electron and $\gamma$ is the Lorentz factor of the slice. Note that the charge density in each slice is assumed to be uniform.

For the calculation of the transverse space charge field, each segmentation slice is first assumed to be a small piece of a pencil beam on the $z$-axis, for example, from $z_{j}$ to $z_{j+1}$ 
in Fig. 1. The transverse field can be calculated as the sum of the fields from each piece of pencil beam and these fields can be calculated analytically by integrating space charge fields over $z$ : [13]

$$
\begin{aligned}
E_{r \text {.pencil }}(r, z)= & \frac{Q}{4 \pi \epsilon_{0} L r}\left(\frac{z-L / 2}{\sqrt{(z-L / 2)^{2}+\frac{r^{2}}{\gamma^{2}}}}\right. \\
& \left.-\frac{z+L / 2}{\sqrt{(z+L / 2)^{2}+\frac{r^{2}}{\gamma^{2}}}}\right),
\end{aligned}
$$

where $Q$ is the charge of the piece of the pencil beam, $L$ is the length of the piece of the pencil beam and $r$ and $z$ are the position of the field's calculation point, where the center of the piece of the pencil beam is located at $z=0$. The forms of Eqs. (1) and (2) are also the same as those used in Ref. [10].

In practice, however, each segmentation piece is not a pencil beam but a cylindrical slice. Here, the transverse field correction function $F_{c}$ is defined to simplify the field calculation. The field from each cylindrical slice can be derived by multiplying the field from the pencil beam by $F_{c}$ as follows: [13]

$$
E_{r \cdot \text { cylinder }}(r, z)=F_{c}\left(\frac{r}{R_{S}}, \frac{2 r}{\gamma L}, \frac{z}{L}\right) \cdot E_{r \cdot \text { pencil }}(r, z),
$$

where $E_{r \text {.cylinder }}$ and $E_{r \text {.pencil }}$ are transverse space charge fields and $F_{c}$ is the transverse correction function.

$F_{c}$ is a function of three variables, as shown in Eq. (3). Among these variables, the variation of $F_{c}$ with $z / L$ can be calculated simply by linear interpolation. By preparing two-dimensional mapping data as data files and loading the data files, the transverse space charge fields $E_{r \text {.cylinder }}$ can be calculated numerically.

Note that the radius of the slice defined here is not $\left(r_{i j}+r_{i \cdot j+1}\right) / 2$ but $\left(R_{j}+R_{j+1}\right) / 2$ even for field calculations for electrons located inside the beam. The correction function is also valid for the electrons inside the beam.

\section{NEW BEAM-TRACKING SIMULATION CODE}

The space charge field calculation scheme for the new beam-tracking code is the same as that for the multiple beam envelope equations. To remove the assumption that each slice is rigid, several improvements have been added to the new code. The new features of the new code are as follows: (i) A hollow cylinder is considered instead of segmentation slices in order to model a warped slice. Space charge fields are calculated from each hollow cylinder. (ii) Longitudinal space charge fields at off-axis positions are newly calculated to accurately calculate the trajectory of electrons at off-axis positions. (iii) Motion equations of electrons are solved by the fourth-order Runge-Kutta

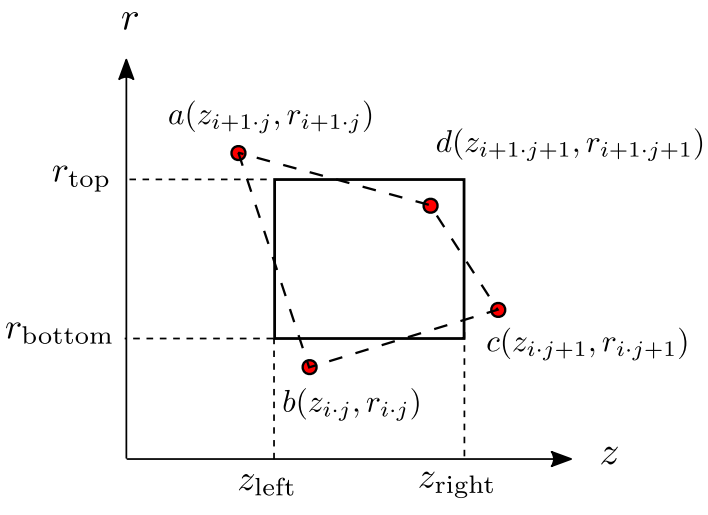

FIG. 2. Longitudinal section of a hollow cylinder used in the new code.

method instead of solving the envelope equations to avoid complicated forms of the envelope equations.

\section{A. Modeling bunched beam using hollow cylinders}

A schematic profile of a hollow cylinder is shown in Fig. 2. The rectangle illustrated at the center of Fig. 2 is a longitudinal section of one of a hollow cylinder and the red circles are tracked electrons. The quadrilateral, that is formed by electrons $a, b, c$ and $d$ is not a rectangle in the usual case, although the section of the hollow cylinder is assumed to be a rectangle by the following process:

$$
\left\{\begin{array}{l}
r_{\text {top }}=\left(r_{i+1 \cdot j}+r_{i+1 \cdot j+1}\right) / 2 \\
r_{\text {bottom }}=\left(r_{i \cdot j}+r_{i \cdot j+1}\right) / 2 \\
z_{\text {left }}=\left(z_{i+1 \cdot j}+z_{i \cdot j}\right) / 2 \\
z_{\text {right }}=\left(z_{i+1 \cdot j+1}+z_{i \cdot j+1}\right) / 2,
\end{array}\right.
$$

where $r_{\text {top }}, r_{\text {bottom }}, z_{\text {left }}$ and $z_{\text {right }}$ are the boundary positions of the hollow cylinder. Note that $z_{i \cdot j}$ is different from $z_{i+1 \cdot j}$ in the new code since the slice can warp.

Figure 3 shows a schematic image of modeling a warped slice using hollow cylinders in the new code. The dark colored parts are longitudinal sections of real hollow cylinders and the light colored parts are virtual slices used for the space charge field calculations mentioned below.

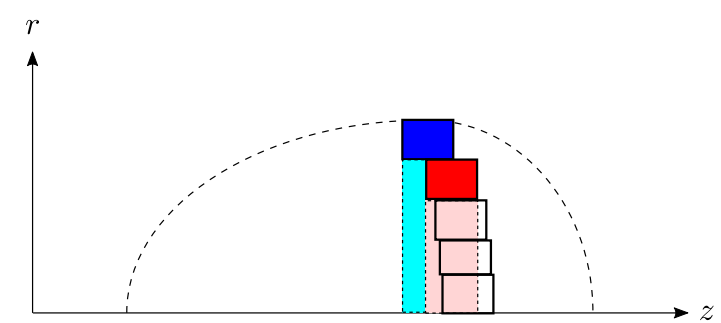

FIG. 3. Modeling a warped slice in a bunched beam using hollow cylinders. The dashed line indicates the entire bunched beam. 
The warped slice is composed of several hollow cylinders that are shifted longitudinally.

The space charge field produced by each hollow cylinder must be calculated in the new code. Here, the space charge field produced by the dark red hollow cylinder in Fig. 3 is considered. The field produced by the hollow cylinder can be calculated by subtracting the field produced by the light red virtual slice from the field produced by the virtual slice composed of the dark and light red parts. For the other hollow cylinders such as the blue parts, the same process can be applied. Note that the charge in a hollow cylinder is constant throughout a beam-tracking calculation and the charge density in a hollow cylinder is assumed to be uniform. The charge density in the light colored virtual slice is also uniform and the same as that in the corresponding hollow cylinder.

\section{B. Calculation of longitudinal space charge fields at off-axis position}

The calculation scheme of transverse space charge fields produced by a slice in the new code is the same as that in the multiple beam envelope equations. Here, the calculation of longitudinal space charge fields produced by a slice at an off-axis position is described.

For the longitudinal space charge field at the off-axis position $r_{0}$, the field $E_{z \text { pencil }}\left(r, z_{0}\right)$ produced by a small piece of a pencil beam, which is illustrated as a red line in the left figure of Fig. 4, is first calculated. The off-axis field produced by a slice, $E_{z \text { slice }}\left(r_{0}, z_{0}\right)$, at position $z_{0}$ apart from the slice center can be derived numerically by integrating the field produced by a pencil beam, $E_{z \text { pencil }}\left(r, z_{0}\right)$. In the subsequent discussion, the width of a slice is defined as $L$, the radius as $R_{s}$ and the charge as $Q$.

$E_{z \text { :pencil }}\left(r, z_{0}\right)$ can be derived as follows. An electric field in a laboratory frame, which is produced by an electron with linear uniform motion and velocity $\boldsymbol{v}$, is generally given as

$$
\boldsymbol{E}(\boldsymbol{R})=\frac{1}{4 \pi \epsilon_{0} \gamma^{2}} \frac{-e \boldsymbol{R}}{\left[|\boldsymbol{R}|^{2}-\frac{|\boldsymbol{v} \times \boldsymbol{R}|^{2}}{c^{2}}\right]^{3 / 2}},
$$

where $\boldsymbol{R}$ is the vector from the present position of the electron. For a uniformly charged pulsed pencil beam with length $L$, line charge $\rho=Q / \pi R_{s}^{2}$ and energy $\gamma m_{0} c^{2}$, as shown in Fig. 5, $d E_{z}$ at point $O\left(z_{0}, r\right)$ produced by the

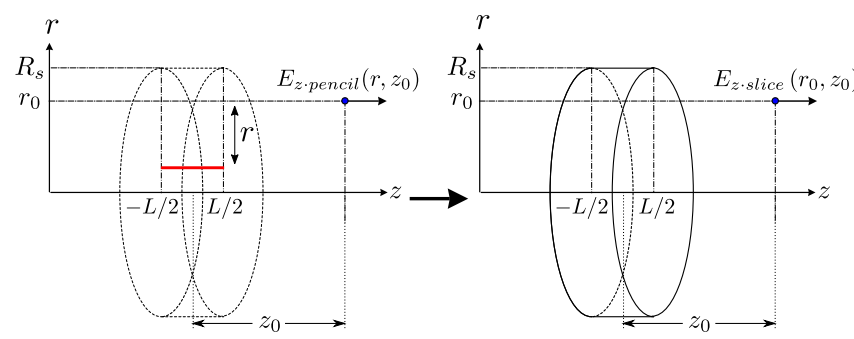

FIG. 4. Calculation model of longitudinal space charge field.

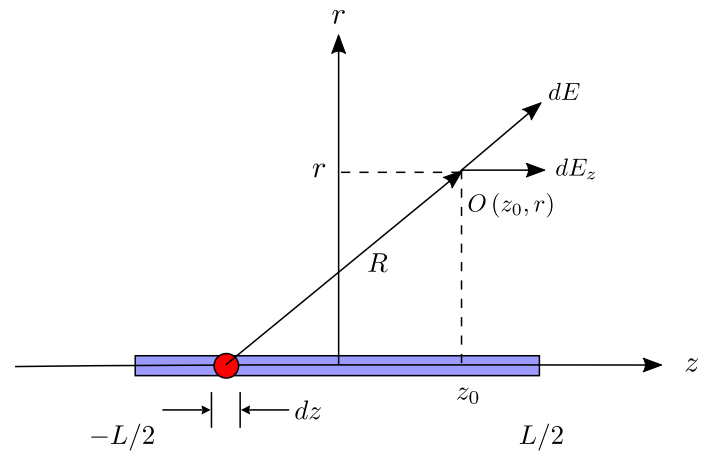

FIG. 5. Calculation model of longitudinal space charge field produced by a pencil beam.

charges located within width $d z$ is calculated from Eq. (5) as follows:

$$
d E_{z}=-\frac{1}{4 \pi \epsilon_{0} \gamma^{2}} \cdot \frac{\rho}{L} d z \cdot \frac{z_{0}-z}{\left[\left(z_{0}-z\right)^{2}+\frac{r^{2}}{\gamma^{2}}\right]^{3 / 2}}
$$

$E_{z \cdot \text { pencil }}\left(r, z_{0}\right)$ is derived by integrating Eq. (6) over $z$ :

$$
\begin{aligned}
& E_{z \cdot \text { pencil }}\left(r, z_{0}\right) \\
& =\int_{-L / 2}^{L / 2} d E_{z}=-\frac{\rho}{4 \pi \epsilon_{0} \gamma^{2} L^{2}} \\
& \quad \times\left[\frac{1}{\sqrt{\left(\frac{z_{0}}{L}-\frac{1}{2}\right)^{2}+\left(\frac{r}{\gamma L}\right)^{2}}}-\frac{1}{\sqrt{\left(\frac{z_{0}}{L}+\frac{1}{2}\right)^{2}+\left(\frac{r}{\gamma L}\right)^{2}}}\right] .
\end{aligned}
$$

$E_{z \text { slice }}\left(r_{0}, z_{0}\right)$ can be calculated by numerically integrating Eq. (7) over $\theta$ and $r$ as shown in Fig. 4.

$E_{z \cdot \text { slice }}\left(r_{0}, z_{0}\right)=\int_{-r_{0}}^{R_{s}-r_{0}} \int_{0}^{2 \pi} E_{z \cdot \text { pencil }}\left(r, z_{0}\right) \cdot\left(r+r_{0}\right) d \theta d r$.

From Eqs. (7) and (8), $E_{z \text { :slice }}\left(r_{0}, z_{0}\right)$ is a function of $2 r_{0} / \gamma L$ and $z_{0} / L$. On the other hand, $E_{z \text { slice }}\left(r_{0}, z_{0}\right)$ is clearly a function of $r_{0} / R_{s}$ from the geometric layout. Therefore, $E_{z \text { slice }}\left(r_{0}, z_{0}\right)$ is a function of three independent variables, $r_{0} / R_{s}, 2 r_{0} / \gamma L$ and $z_{0} / L$. By preparing threedimensional mapping data, we can correctly calculate the longitudinal space charge fields at off-axis positions by loading the mapping data into the calculation process. Note that the description $2 r_{0}$ is meaningful for comparison with $\gamma L[13]$.

\section{CALCULATIONS OF BEAM DYNAMICS USING NEW BEAM-TRACKING SIMULATION CODE}

Here, examples of beam dynamics calculations using the new code are shown, which could not be accurately 
TABLE I. Parameters for calculations of beam dynamics in the BNL-type rf gun cavity.

Laser length

Laser spot size

Charge per bunch for Figs. 6-8

Maximum electric field on the cathode

Energy at the exit of the cavity

Initial rf phase (when the head of the bunch is emitted)

Initial emittance

Segmentation numbers for the new code

Segmentation numbers for the multiple beam envelope equations

Number of particles for the existing code

calculated using the multiple beam envelope equations in previous studies $[13,14]$.

\section{A. Calculations of beam dynamics in BNL-type rf gun cavity}

The beam dynamics in the 1.6 cell BNL-type rf gun cavity is calculated using the multiple beam envelope equations. The calculation domain includes only an $\mathrm{rf}$ gun cavity with image charge effects at a cathode and the subsequent free space. Initial calculation parameters are shown in Table I. The initial thermal emittance is not taken into account; therefore, it is assumed to be $0 \mathrm{mrad}$. The cathode of the cavity is located at $z=0$.

Figure 6 shows the calculation results at $z=0.15 \mathrm{~m}$ after the rf gun cavity with a charge of $1.0 \mathrm{nC} /$ bunch calculated using the multiple beam envelope equations, along with those calculated by the existing threedimensional particle-tracking simulation code [9], which was developed by the author. These results are also shown in Ref. [13]. In the existing code, the point-to-point calculation technique is used for the space charge field calculation. That is, actions between every pair of particles are calculated. For the tracking of particles, the equations of motion of the particles are solved by the fourth-order Runge-Kutta method. The number of particles used in the existing three-dimensional code is $1 \times 10^{5}$. Initial particles are aligned using Hammersley's sequence [15] to reduce the random noise caused by the distribution of particle positions. In the multiple beam envelope equations, the segmentation numbers $m$ and $n$ are both 10 .

Figure 6(a) shows the bunch shapes at $z=0.15 \mathrm{~m}$. Each dot on the solid lines is an electron traced using the multiple beam envelope equations. The clouds of small dots are the particles calculated using the existing code. The particles are color-coded according to the initial segmentation slices used in the multiple beam envelope equations.

Figure 6(b) shows the energy distributions in the bunch at $z=0.15 \mathrm{~m}$. The black dots on the line are obtained

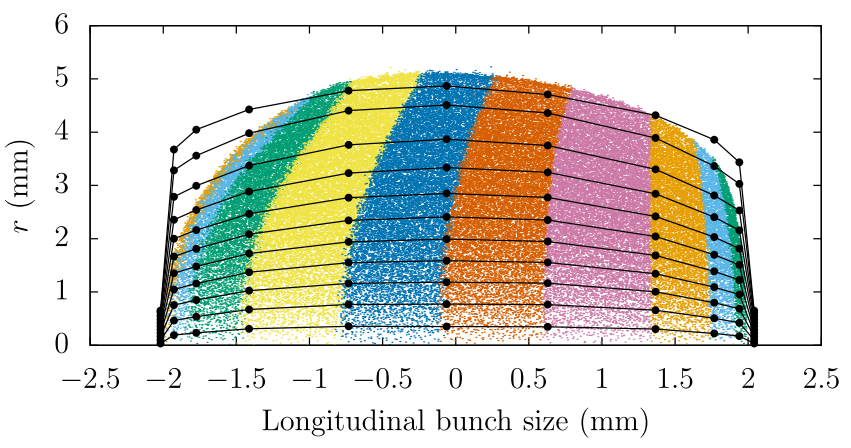

(a) Bunch shapes after the rf gun cavity.

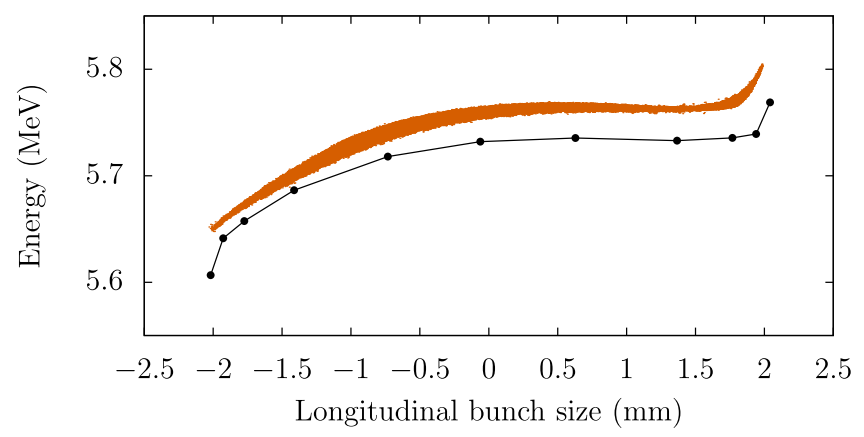

(b) Energy distributions after the rf gun cavity.

FIG. 6. Beam dynamics calculated using the multiple beam envelope equations for a charge of $1.0 \mathrm{nC}$ per bunch in the BNL-type rf gun cavity.

using the multiple beam envelope equations, and the clouds of small red dots are the results of the existing code.

In Fig. 6(a), each slice calculated by the existing code is warped because the charge density is high. Therefore, the multiple beam envelope equations cannot be used to calculate the bunch shape correctly in this case.

Figure 7 shows the calculation results obtained using the new code and the existing code under the same conditions as those shown in Fig. 6. $m$ and $n$ are both 20 in the new code. The calculation scheme of the image charge effects for the new code is the same as that for the multiple beam envelope equations [13]. In Fig. 7(a), each slice calculated by the new code becomes warped, and the shape of the bunch is calculated correctly. In Fig. 7(b), the clouds of small black dots indicate the results calculated by the new code. Because space charge fields at off-axis positions are correctly calculated in the new code, the energy distributions are not plotted as a single line but as a shape having an area.

Time evolutions of the normalized emittance are shown in Fig. 8, where the emittance is defined as

$$
\epsilon_{r} \equiv\langle\gamma\rangle\langle\beta\rangle \sqrt{\left\langle r^{2}\right\rangle\left\langle r^{\prime 2}\right\rangle-\left\langle r \cdot r^{\prime}\right\rangle^{2}} .
$$

The emittance calculated by the multiple beam envelope equations is slightly different from that calculated using the existing code because the segmentation slices are rigid. The 


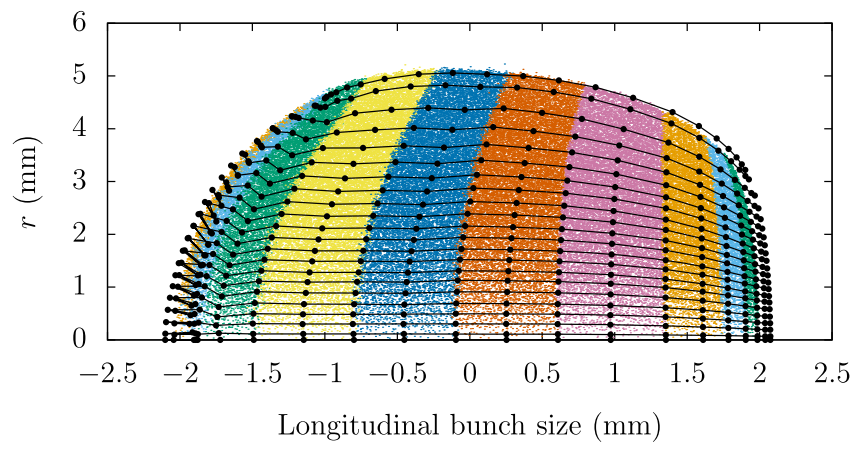

(a) Bunch shapes after the rf gun cavity.

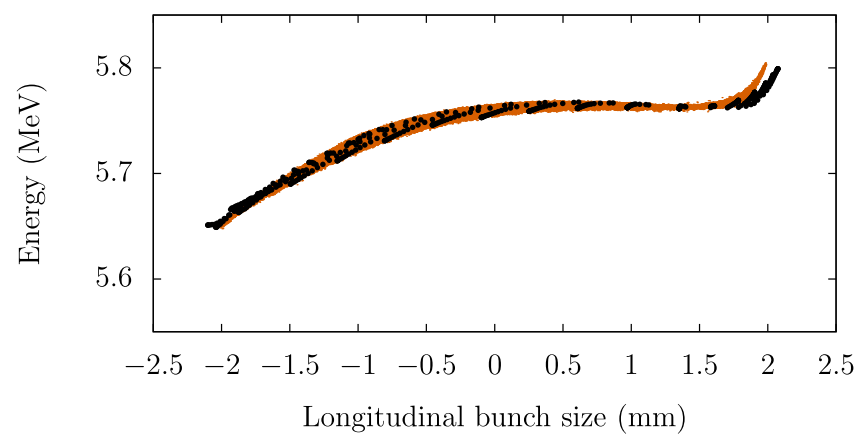

(b) Energy distributions after the rf gun cavity.

FIG. 7. Beam dynamics calculated using the new code for a charge of $1.0 \mathrm{nC}$ per bunch in the BNL-type rf gun cavity.

emittance obtained by the new code is almost the same as that obtained by the existing code.

Figure 9 shows the calculated emittance at $z=0.15 \mathrm{~m}$ as a function of the charge per bunch. The author mentioned in Ref. [13] that the applicable range of the multiple beam envelope equations is under $0.5 \mathrm{nC} /$ bunch for this case because the calculated emittance becomes different from that calculated by the existing code. Because the slice can warp and the bunch shape is calculated correctly using the new code, the emittances calculated by the new code are almost the same as those calculated by the existing code even when the charge is greater than $0.5 \mathrm{nC} /$ bunch.

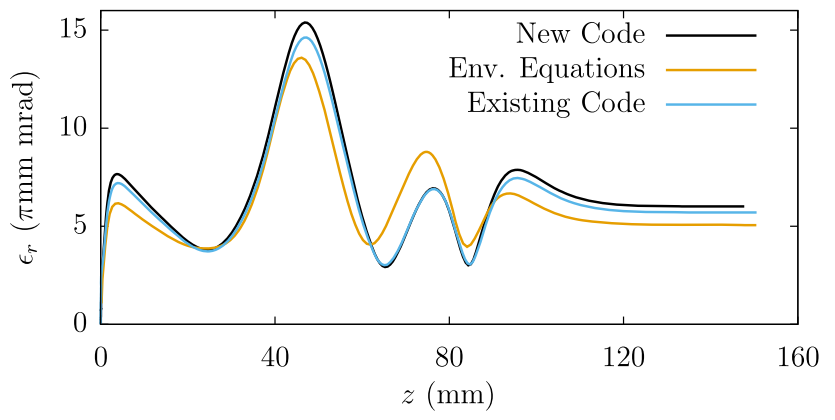

FIG. 8. Emittance evolutions for a charge of $1.0 \mathrm{nC}$ per bunch in the BNL-type rf cavity. The rf gun cavity structure is located from $z=0$ to $77.67 \mathrm{~mm}$.

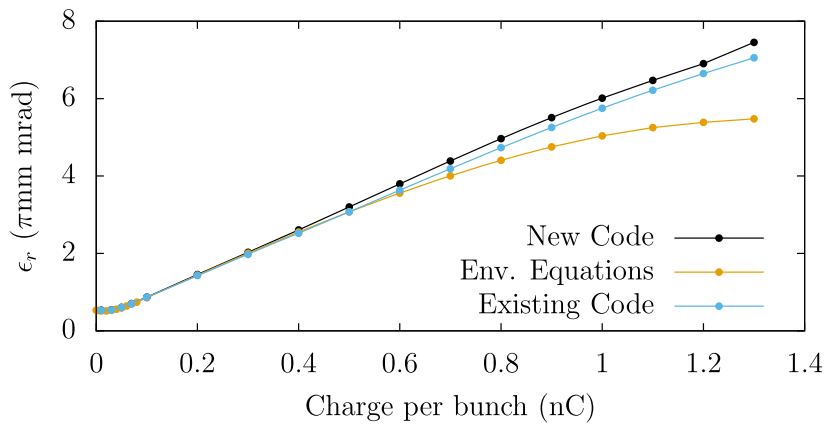

FIG. 9. Dependence of emittance on charge per bunch.

\section{B. Calculations of beam dynamics involving focusing by solenoidal coils}

The calculation for the SPring- 8 rf gun injector system is performed using the multiple beam envelope equations [14] and the new code. This system consists of a single-cell S-band rf gun cavity with a copper cathode [16], two solenoidal coils after the rf gun cavity and a 3-m long traveling wave accelerator structure whose entrance is located $1.4 \mathrm{~m}$ from the cathode surface. The beam energy is $3.7 \mathrm{MeV}$ at the exit of the rf gun cavity and $30.0 \mathrm{MeV}$ at the exit of the accelerator structure. The calculation parameters are summarized in Table II.

Figure 10 shows the calculated transverse rms beam size from the cathode $(z=0)$ to the exit of the accelerator structure. The beams are defocused at exit of the rf gun cavity and focused by the solenoidal coils. The waists of the beams appear in the accelerator structure at about $z=2.5 \mathrm{~m}$. The time evolutions of the beam sizes are almost the same among the three methods.

Figure 11 shows the results calculated by the multiple beam envelope equations, along with those calculated by the existing code. The bunch shapes and energy distributions at $z=4.5 \mathrm{~m}$, which is after the waist, do not agree with each other. In Fig. 11(a), the solid lines in the

TABLE II. Parameters for calculations of beam dynamics in the SPring-8 rf gun injector system with focusing fields.

\begin{tabular}{lr}
\hline \hline Laser length & $\begin{array}{r}20 \mathrm{ps} \text { uniform } \\
\phi 1.2 \mathrm{~mm} \\
\text { uniform } \\
50 \mathrm{pC}\end{array}$ \\
Laser spot size & $\begin{array}{r}57.0 \mathrm{MV} / \mathrm{m} \\
\text { Sin } 5 \mathrm{deg} .\end{array}$ \\
$\begin{array}{l}\text { Maximum electric field on the cathode } \\
\text { Initial rf phase (when the head of the bunch is } \\
\quad \text { emitted) }\end{array}$ & $\begin{array}{r}0 \mathrm{mrad} \\
\text { Initial emittance }\end{array}$ \\
$\begin{array}{l}\text { Entrance of an accelerator structure } \\
\text { Energy at the exit of a cavity }\end{array}$ & $3.7 \mathrm{MeV}$ \\
Energy at the exit of an acc. structure & $m=.0 \mathrm{MeV}$ \\
Segmentation numbers for the new code & $n=20$ \\
Segmentation numbers for the multiple beam & $m=n=10$ \\
$\quad$ envelope equations & \\
Number of particles for the existing code & $4 \times 10^{4}$ \\
\hline \hline
\end{tabular}




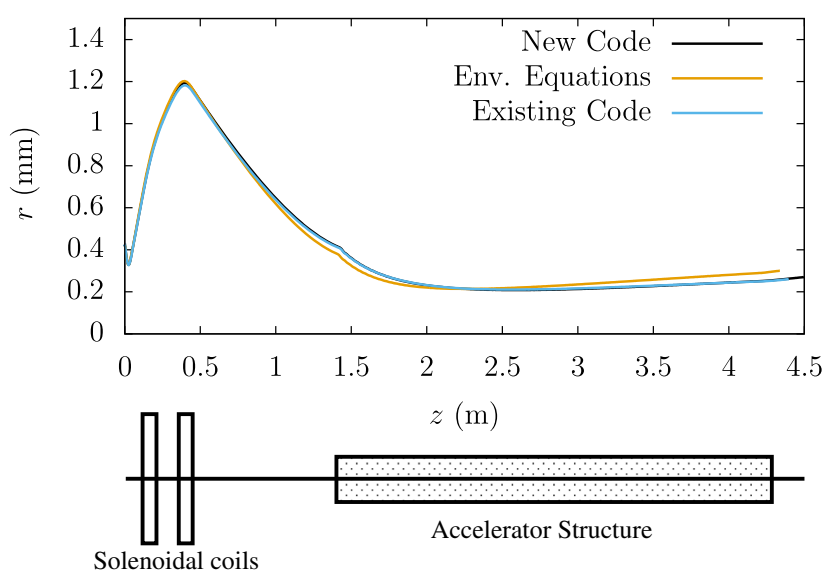

FIG. 10. Time evolutions of transverse rms beam size for the SPring-8 rf gun injector system with focusing fields.

calculated bunch shape, which represents the particle positions and are initially in order similarly to those in Fig. 1, intersect when they pass the waist.

The charge density of the segmentation slices used to calculate space charge fields is assumed to be uniform in the multiple beam envelope equations, as mentioned in Sec. II. In practice, however, transverse variation of the charge density occurs when electrons intersect. Therefore, the intersections cannot be modeled by these uniform

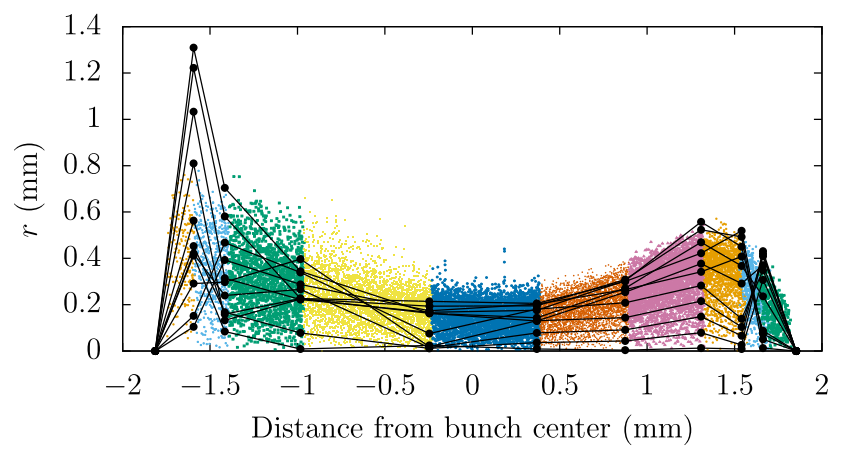

(a) Bunch shapes at the exit of the accelerator structure.

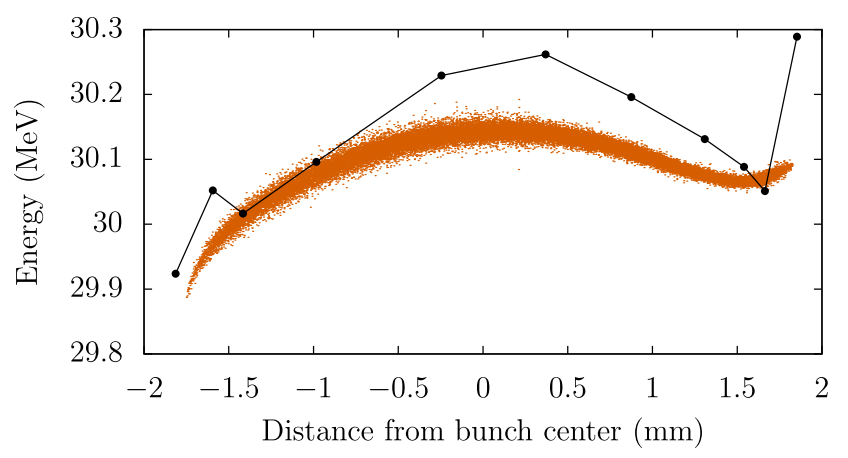

(b) Energy distributions at the exit of the accelerator structure.

FIG. 11. Beam dynamics for the SPring- 8 rf gun injector system with focusing fields calculated by the multiple beam envelope equations.

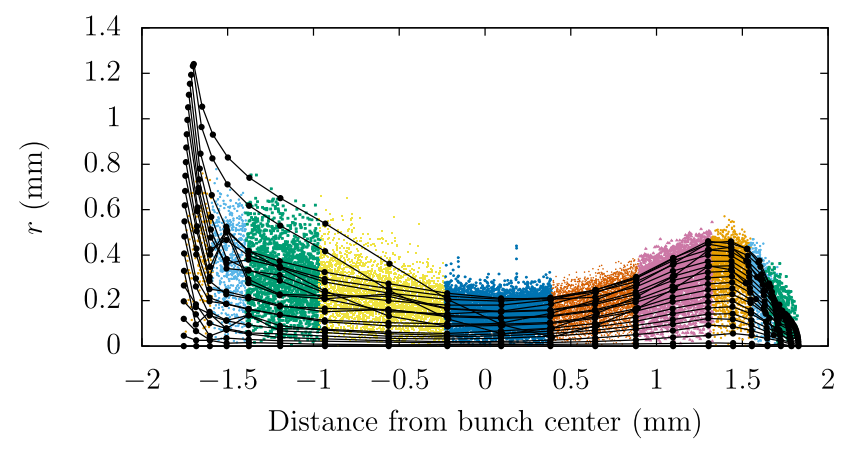

(a) Bunch shapes at the exit of the accelerator structure.

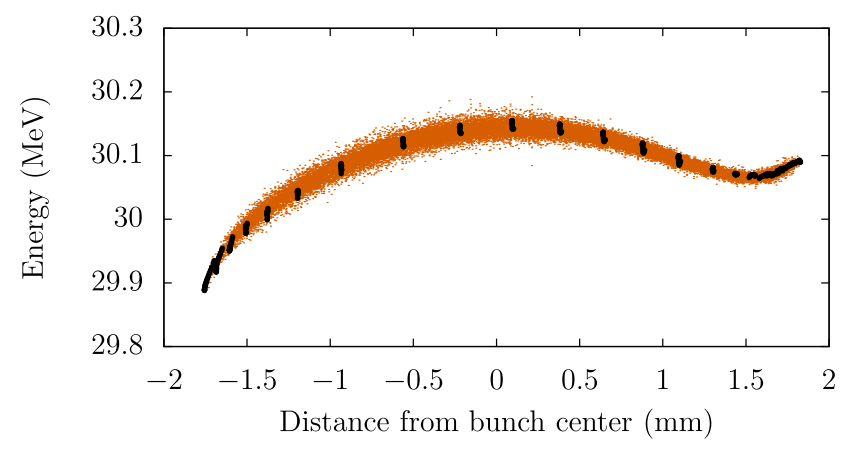

(b) Energy distributions at the exit of the accelerator structure.

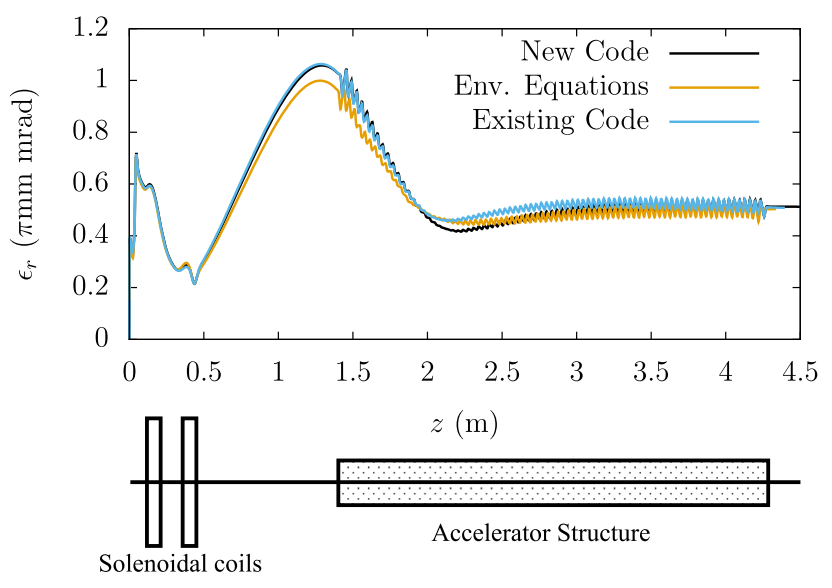

(c) Time evolutions of emittance.

FIG. 12. Beam dynamics for the SPring-8 $\mathrm{rf}$ gun injector system with focusing fields calculated by the new code.

slices. Consequently, the bunch shape and energy distribution cannot be calculated correctly.

Figure 12 shows results calculated by the new code. The solid lines in the bunch shown in Fig. 12(a) also intersect after the waist. A schematic profile of the hollow cylinder in the new code when the solid lines $a d$ and $b c$ intersect is shown in Fig. 13. The hollow cylinder is formed by the quadrilateral abcd when there are no intersections, as shown in Fig. 2. On the other hand, when the intersection occurs in Fig. 13, the hollow cylinder is formed by the quadrilateral $a b d c$ while maintaining the charge in the quadrilateral $a b c d$ in Fig. 2. A hollow cylinder has the possibility of overlapping with another hollow cylinder 


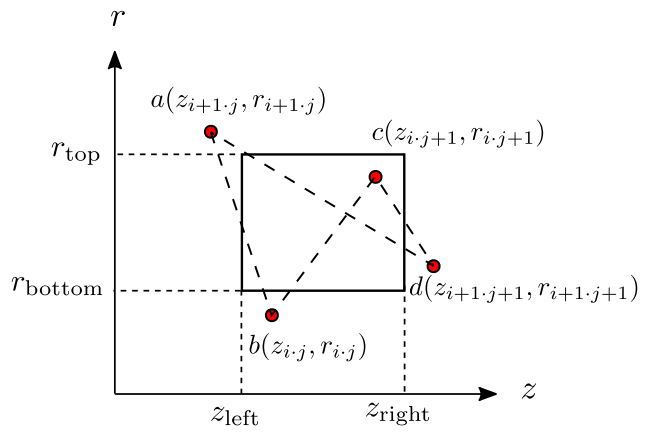

FIG. 13. Longitudinal section of a hollow cylinder used in the new code when intersection occurs.

when intersections occur; however, these overlaps are useful for modeling the transverse variation of the charge density. Therefore, the fields produced by each hollow cylinder and also the bunch shape can be calculated correctly. Consequently, the energy distribution shown in Fig. 12(b) and the emittance shown in Fig. 12(c) agree with those calculated by the existing code.

\section{Accuracy of emittance}

Other examples of calculation for the SPring- $8 \mathrm{rf}$ gun injector system by the new code and the existing code are shown here. The calculation parameters are slightly different from those used in Sec. IV B and are shown in Table III.

The strength of the focusing field is the same as that for the case described in Sec. IV B and the waist of the beam is formed at the exit of the accelerator structure, as shown in Fig. 14. In these examples, the calculation parameters are optimized to realize ultralow emittance at the exit of the accelerator structure, as shown in Fig. 15. Therefore, the emittance dependence on the number of particles, which is caused by the random noise of space charge fields formed by the particle position in the existing code, becomes strong. The black dots in Fig. 16(a) show the emittance dependence on the segmentation number in the new code and those in Fig. 16(b) show the emittance dependence on the number of particles in the existing code. In the new code, space charge fields are produced not by a point charge but by a hollow

TABLE III. Parameters for low-emittance beam dynamics calculations in the SPring-8 rf gun injector system.

Laser length

Laser spot size

Charge per bunch

Maximum electric field on the cathode

Initial rf phase (when the head of the bunch is emitted)

Initial emittance

Entrance of the accelerator structure

Energy at the exit of the cavity

Energy at the exit of the acc. structure

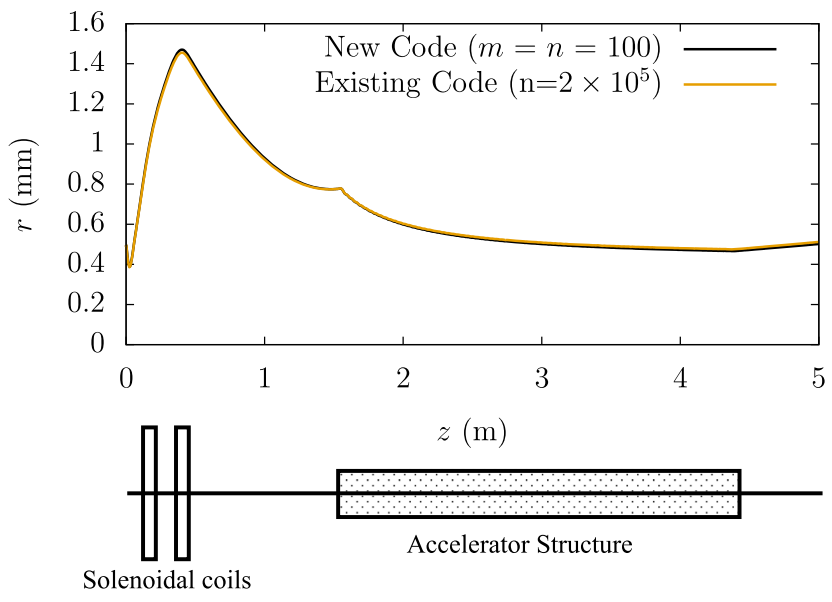

FIG. 14. Time evolutions of transverse rms beam size for lowemittance beam dynamics calculations for the SPring-8 rf gun injector system. The results for the new and existing codes are shown together.

cylinder that has a volume. Therefore, there is no random noise caused by the particle positions, and the emittance dependence in the new code is markedly weaker than that in the existing code. In Fig. 16(b), the result calculated by the new code is plotted at a point where $n$ is infinity. The emittance calculated by the new code is expected to coincide with that calculated by the existing code when the number of particles becomes infinity.

The calculation time of the new code is much shorter than that of the existing code because the segmentation number in the new code is small. Although depending on the type of CPU, the calculation takes 3 weeks for the existing code when the number of particles is $2 \times 10^{5}$ using an 8-core Xeon W5590 3.33 GHz processor. In contrast, the calculation takes $24 \mathrm{~h}$ for the new code when $m=n=100$. In addition, it takes only $3 \mathrm{~min}$ when $m=n=20$, and the calculated emittance is not significantly different from the emittance when $m=n=100$.

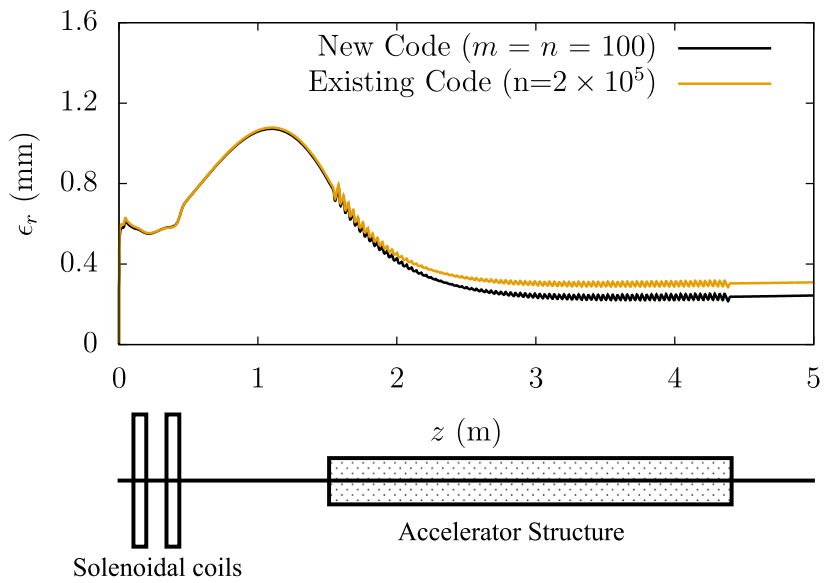

FIG. 15. Time evolutions of emittance for low-emittance beam dynamics calculations for the SPring-8 rf gun injector system. 

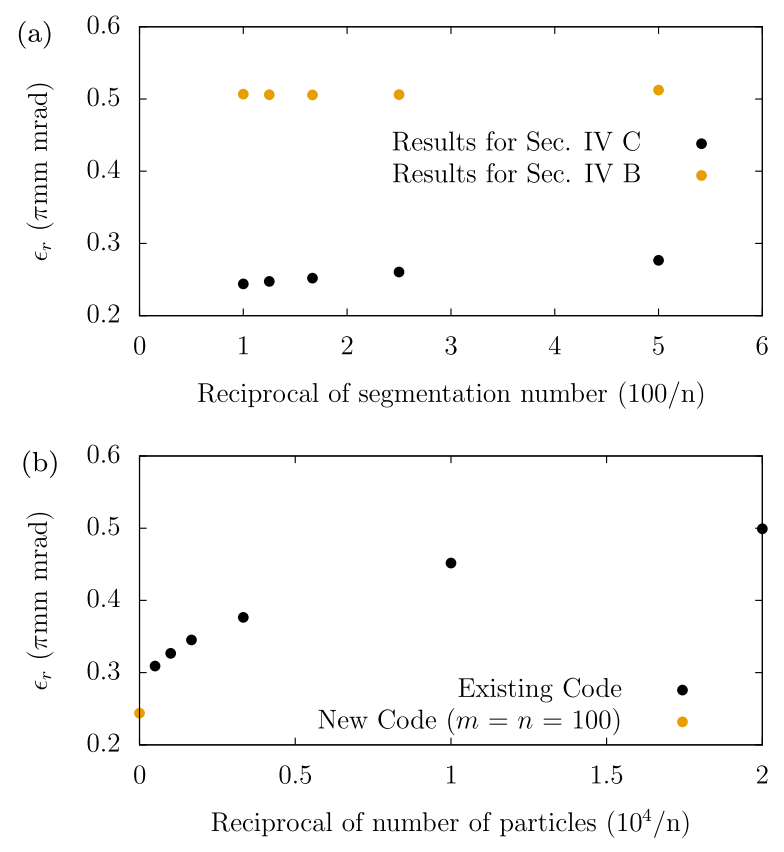

FIG. 16. Emittance dependence on numbers of particles and segmentations. (a) Emittance dependence on segmentation number in the new code. $m$ and $n$ are the same in each calculation. $m=n=20$ for the rightmost points and $m=n=$ 100 for the leftmost points. (b) Emittance dependence on numbers of particles used in the existing code. The number of particles is $5 \times 10^{3}$ for the rightmost point and $2 \times 10^{5}$ for the leftmost black point. A result calculated by the new code whose segmentation numbers are $m=n=100$ is plotted as a color point.

Note that the emittance dependence on the segmentation number is particularly strong in this example. The dependence in the case described in Sec. IV B is almost flat, as shown by the color points in Fig. 16(a).

\section{CONCLUSION}

A novel beam-tracking simulation code using the bulkto-point calculation technique for space charge fields, whose calculation scheme is based on that of the multiple beam envelope equations, was developed.

One limitation of the multiple beam envelope equations, that is, the rigid longitudinal slice, is removed in the new code for the accurate calculation of emittance.

Two examples that cannot be accurately calculated by the multiple envelope equations are calculated by the new code. One is the beam dynamics in the BNL-type rf gun cavity with high charge and the other is that in the SPring- 8 rf gun injector system, which has solenoidal coils for beam focusing. The two examples are accurately calculated by the new code.

An example of the SPring-8 $\mathrm{rf}$ gun injector system with optimum parameters for ultralow emittance is calculated by the new and existing codes to confirm the accuracy of the calculated emittance. Because the calculated space charge fields are not produced by a point charge but by a hollow cylinder having a volume, the emittance dependence on the segmentation number calculated using the new code is much weaker than that on the number of particles calculated using the existing code. Therefore, the calculation time is much shorter, and the emittance calculated by the new code is expected to coincide with that calculated by the existing code when the number of particles becomes infinity.

This newly developed simulation code will be useful for calculating low-emittance electron injectors because of the rapid and accurate calculation of emittance.

\section{ACKNOWLEDGMENTS}

The author wish to thank Dr. H. Hanaki, JASRI/SPring8 , for detailed discussions on the scheme of the new beamtracking simulation code.

[1] J. D. Lawson, The Physics of Charged-Particle Beams (Oxford Science Publications, New York, 1988).

[2] K. J. Kim, Nucl. Instrum. Methods Phys. Res., Sect. A 275, 201 (1989).

[3] L. Serafini and J. B. Rosenzweig, Phys. Rev. E 55, 7565 (1997).

[4] C. K. Allen and M. Reiser, Phys. Rev. E 55, 7591 (1997).

[5] http://www.pulsar.nl/gpt.

[6] G. Pöplau and K. Flöttmann, in Proceedings of the Tenth European Particle Accelerator Conference, Edinburgh, Scotland (EPS-AG, Edinburgh, Scotland, 2006), p. 2203.

[7] L. M. Young and J. H. Billen, Los Alamos National Laboratory Report No. LA-UR-96-1835, 2004.

[8] J. Qiang, S. Lidia, R. D. Ryne, and C. Limborg-Deprey, Phys. Rev. Accel. Beams 9, 044204 (2006).

[9] A. Mizuno, T. Asaka, H. Dewa, T. Kobayashi, S. Suzuki, T. Taniuchi, H. Tomizawa, K. Yanagida, H. Hanaki, and M. Uesaka, Nucl. Instrum. Methods Phys. Res., Sect. A 528, 387 (2004).

[10] M. Ferrario, A. Mosnier, L. Serafini, F. Tazzioli, and J.-M.Tessier, Particle Accelerators (Overseas Publishers Association, The Netherlands, 1996), Vol. 52, pp. 1-30.

[11] R.Talman, Phys. Rev. Accel. Beams 7, 100701 (2004).

[12] L. G. Vorobiev and R. C. York, in Proceedings of the 18th Particle Accelerator Conference, New York, 1999 (IEEE, New York, 1999), p. 2781.

[13] A. Mizuno, H. Dewa, T. Taniuchi, H. Tomizawa, H. Hanaki, and E. Hotta, Phys. Rev. Accel. Beams 15, 064201 (2012).

[14] A. Mizuno, H. Dewa, T. Taniuchi, H. Tomizawa, H. Hanaki, and E. Hotta, in Proceedings of FEL2012, Nara, Japan (SPring-8 and Kyoto University, Nara, Japan 2012), p. 157.

[15] J. Gonichon, S. C. Chen, L. C-L. Lin, and R. J. Temkin, in Proceedings of the 1993 Particle Accelerator Conference, Washington, DC (IEEE, New York, 1993), p. 2696.

[16] T. Taniuchi, T. Asaka, H. Dewa, T. Kobayashi, A. Mizuno, S. Suzuki, H. Tomizawa, K. Yanagida, and H. Hanaki, in Proceedings of the 21st International Linac Conference, Gyeongju, Korea, 2002 (Pohang Accelerator Laboratory, Pohang, Korea, 2002), p. 683. 